\title{
ОПТИМИЗАЦИЯ КОНФИГУРАЦИИ ЭЛЕКТРОДИНАМИЧЕСКОГО ОБЪЕКТА С ТРЕБУЕМЫМИ ХАРАКТЕРИСТИКАМИ РАССЕЯНИЯ
}

\author{
Преображенский А.П., Чопоров О.Н.
}

В работе рассматривается задача оптимизачия характеристик рассеяния электромагнитных волн на периодической электродинамической структуре. Решение задачи рассеяния осуществляется на основе метода интегральных уравнений, оптимизация характеристик проводится на основе генетического алгоритма. Даны рекомендации по параметрам периодической структуры при заданных уалах наблюдения.

Ключевые слова: дифракция; интегральное уравнение; рассеяние радиоволн; радиосвязь; дифракционная структура; оптимизациия; генетический алгоритм.

\section{THE OPTIMIZATION OF ELECTRODYNAMIC CONFIGURATION OBJECT WITH THE DESIRED CHARACTERISTICS OF SCATTERING}

\section{Preobrazhensky A.P., Choporov O.N.}

This paper considers the problem of optimization of the characteristics of scattering of electromagnetic waves on periodic electrodynamic structure. The solution of the scattering problem is based on the method of integral equations, the optimization of the characteristics is based 
on the genetic algorithm. Recommendations on the parameters of the periodic structure under given angles are given.

Keywords: diffraction; integral equation; scattering of radio waves; telecommunication; diffraction structure; optimization; genetic algorithm.

Задачи рассеяния электромагнитных волн встречаются во многих практических приложениях, в том числе и при проектировании систем радиосвязи [1-5].

При математическом моделировании объекты представляются как совокупность жестко связанных отражателей, имеющих определенные параметры.

В ряде случаев мы можем использовать интегральные уравнения для того, чтобы исследовать периодические структуры, в том числе для дифракционных решеток, образованных бесконечно пластинами, которые имеют произвольную форму и идеально проводящие [6].

Задачу, связанную с определением токов на периодической структуре, когда на ней происходит рассеяние электромагнитной волны, мы решали в рамках метода интегральных уравнений [79]. Для одного периода плотности тока есть возможность записи интегрального уравнения [10]

$$
\begin{gathered}
\vec{J}(x, y, z)+2 \cdot \int_{S}\left[\vec{n}(x, y, z) \times\left[\vec{J}\left(x^{\prime}, y^{\prime}, z^{\prime}\right) \times \operatorname{grad} G\left(x, y, z, x^{\prime}, y^{\prime}, z^{\prime}\right)\right]\right] d s= \\
=2 \cdot\left[\vec{n}(x, y, z) \times \vec{H}^{i}(x, y, z)\right],
\end{gathered}
$$

где

$$
\begin{aligned}
& G\left(x, y, z, x^{\prime}, y^{\prime}, z^{\prime}\right)=\exp (-i k r) /(4 \pi r), \\
& r=\sqrt{\left(x-x^{\prime}\right)^{2}+\left(y-y^{\prime}\right)^{2}+\left(z-z^{\prime}\right)^{2}} .
\end{aligned}
$$

Если рассматривать случай одномерной линейной гребенки, то уравнение (1) приводится к бесконечной системе интегральных уравнений. Для одного из элементов гребенки мы назначим нулевой индекс и проведем нумерацию остальных элементов (рис. 1). В этом случае уравнение системы, для которого точка 
наблюдения находится на нулевом объекте, записывается в таком виде

$$
\ldots+\vec{J}_{0}\left(x_{0}, y_{0}, z_{0}\right)+
$$

$+2 \cdot \int_{S_{0}}\left[\vec{n}\left(x_{0}, y_{0}, z_{0}\right) \times\left[\vec{J}_{-1} \times \operatorname{grad} G\left(x_{0}, y_{0}, z_{0}, x_{0}^{\prime}, y_{0}^{\prime}, z_{0}^{\prime}\right)\right]\right] d s+$

$+2 \cdot \int_{S_{1}}\left[\vec{n}\left(x_{0}, y_{0}, z_{0}\right) \times\left[\vec{J}_{1} \times \operatorname{grad} G\left(x_{0}, y_{0}, z_{0}, x_{1}^{\prime}, y_{1}^{\prime}, z_{1}^{\prime}\right)\right]\right] d s+\ldots=$

$$
=2 \cdot\left[\vec{n} \times \vec{H}^{i}\left(x_{0}, y_{0}, z_{0}\right)\right] \text {. }
$$

Для всех элементов структуры можно сказать, что они являются идентичными, и есть отличие по одинаковым точкам элементов лишь в координатах на величину, которая кратна периоду d. Разница в фазах плотностей токов по одинаковым точкам элементов будет $\psi=k d \cos \theta$. В результате, уравнение (1) мы можем свести к следующему интегральному уравнению

$$
\begin{gathered}
\vec{J}_{0}\left(x_{0}, y_{0}, z_{0}\right)+2 \cdot \int_{S_{0}}\left[\vec{n}\left(x_{0}, y_{0}, z_{0}\right) \times\left[\vec{J}_{0} \times\right.\right. \\
\left.\left.\times \sum_{n=-\infty}^{\infty} \exp (i n \psi) \cdot \operatorname{grad} G\left(x_{0}, y_{0}, z_{0}, x_{0}^{\prime}+n d, y_{0}^{\prime}, z_{0}^{\prime}\right)\right]\right] d s= \\
=2 \cdot\left[\vec{n} \times \vec{H}^{i}\left(x_{0}, y_{0}, z_{0}\right)\right] .
\end{gathered}
$$

Этот результат мы можем обобщить для двумерной гребенки. Поскольку система является однородной, то, как и для одномерного случая, разница токов по одинаковым элементам будет лишь в фазах:

$$
\vec{J}_{n l}=\vec{J}_{00} \cdot \exp \left(i n \psi_{1}\right) \cdot \exp \left(i \ell \psi_{2}\right),
$$

где

$$
\psi_{1}=d \cdot \sin \theta \cdot \cos \varphi, \psi_{2}=d \cdot \sin \theta \cdot \sin \varphi .
$$

Обобщение (3) для двумерной гребенки ведет к уравнению

$$
\begin{aligned}
& \vec{J}_{00}\left(x_{00}, y_{00}, z_{00}\right)+2 \cdot \int_{S_{00}}\left[\vec{n}\left(x_{00}, y_{00}, z_{00}\right) \times\left[\vec{J}_{00} \times\right.\right. \\
& \times \sum_{n=-\infty}^{\infty} \sum_{\ell=-\infty}^{\infty} \exp \left(i n \psi_{1}\right) \cdot \exp \left(i \ell \psi_{2}\right) \cdot \operatorname{grad} G\left(x_{00}, y_{00}, z_{00}, x_{00}^{\prime}+\right. \\
&\left.\left.\left.+n d,{y^{\prime}}_{00}+\ell d, z_{00}^{\prime}\right)\right]\right] d S=2 \cdot\left[\vec{n} \times \vec{H}^{i}\left(x_{00}, y_{00}, z_{00}\right)\right] .
\end{aligned}
$$




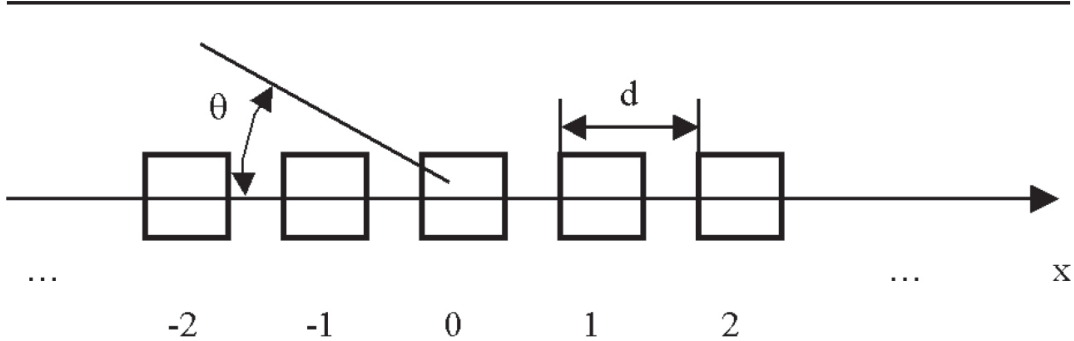

Рис. 1. Одномерная линейная гребенка (вид сверху)

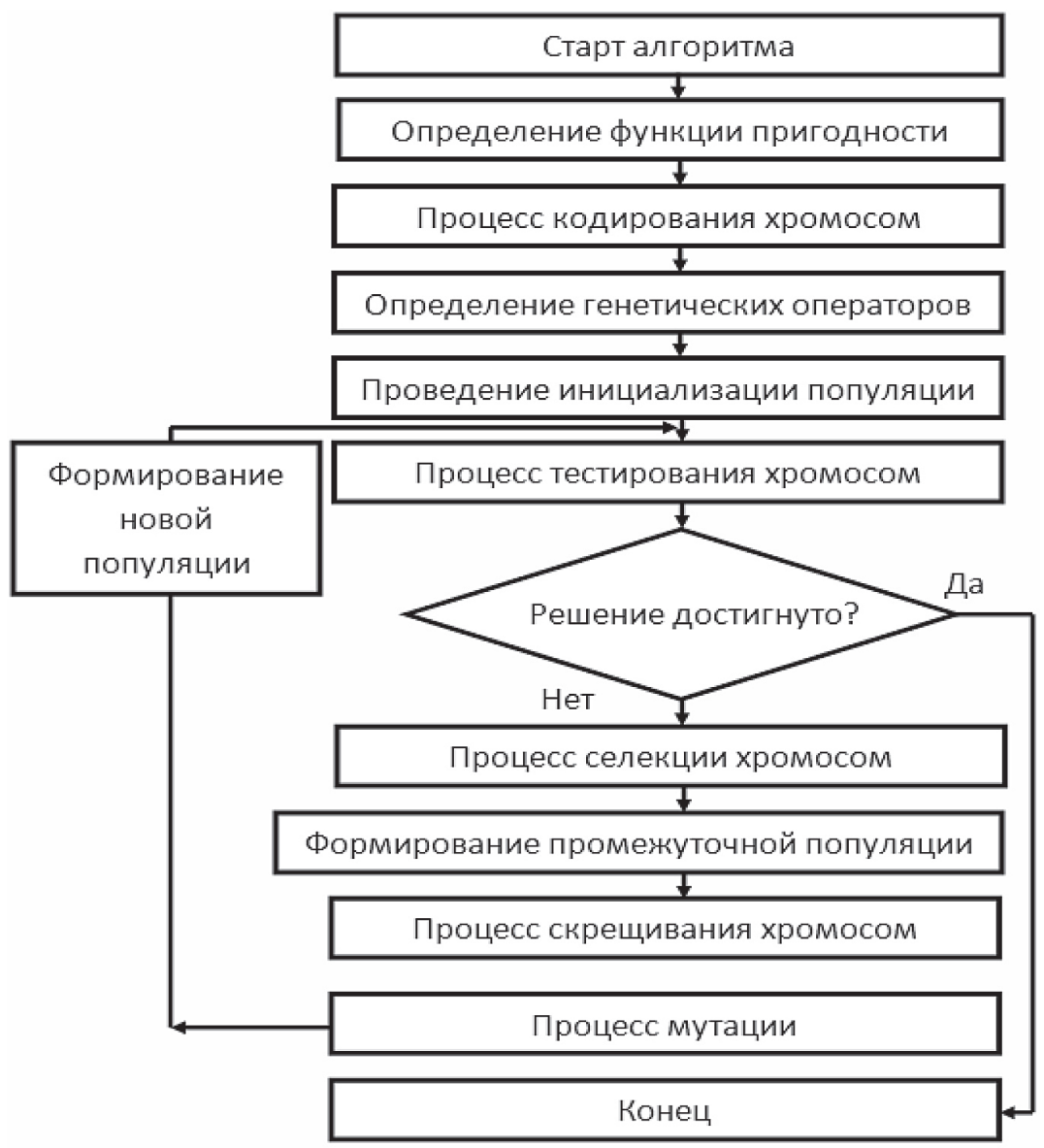

Рис. 2. Схема генетического алгоритма 
Интегральное уравнение решается на основе метода моментов.

Варьируемыми параметрами являются размер элемента и расстояние между элементами.

Для решения задачи, связанной с достижением требуемого уровня характеристик рассеяния гребенки для определенного угла наблюдения мы воспользуемся генетическим алгоритмом (ГА) [12]. Схема ГА приведена на рис. 2.

Хромосома в нашей задаче имеет 4 гена: q, x, с и d. Поскольку ген представляет собой целое число, которое меньше чем 30, то для того, чтобы осуществить кодирование каждого гена мы можем применять 5 битов. В этом случае хромосома представляется в виде, изображенном на рис. 3 .

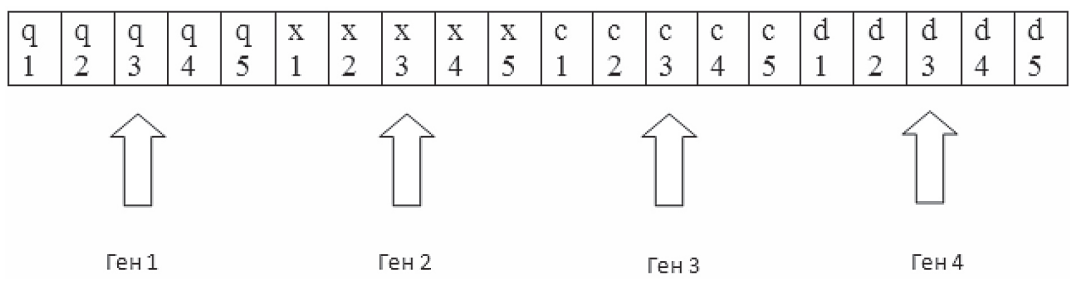

Рис. 3. Схема хромосомы

Например, для того, чтобы сделать поиск решения, мы используем популяцию из 5 хромосом. Сначала осуществим генерацию 5 случайных возможных вариантов решений:

$$
\mathrm{F}(\mathrm{q}, \mathrm{x}, \mathrm{c}, \mathrm{d}), \mathrm{q}, \mathrm{x}, \mathrm{c}, \mathrm{d} \in\{1,2,3 \ldots 30\},
$$

и сделаем связь каждого варианта и ошибки в решении:

$$
\delta=|\mathrm{F}(\mathrm{q}, \mathrm{x}, \mathrm{c}, \mathrm{d})-\mathrm{FD}(\mathrm{q}, \mathrm{x}, \mathrm{c}, \mathrm{d})| .
$$

$\mathrm{B}$ табл. 1 дана демонстрация вариантов решений (используется десятичный код).

Основная идея функционирования ГА состоит в том, чтобы происходило выживание хромосом, которые имеют меньшую ошибку в решении. В этой связи относительная пригодность хромосомы описывается на основе формулы ОП=1/ . 
Таблица 1.

Варианты решения

\begin{tabular}{|c|c|c|c|}
\hline Номер хромосомы & Вариант $(\mathrm{x} 1, \mathrm{x} 2, \mathrm{x} 3, \mathrm{x} 4)$ & Ошибка $\delta$ & ОП \\
\hline 1 & $(1,29,14,4)$ & 82 & 0.013 \\
\hline 2 & $(13,8,1,3)$ & 23 & 0.039 \\
\hline 3 & $(12,4,6,2)$ & 25 & 0.037 \\
\hline 4 & $(22,8,15,18)$ & 131 & 0.0071 \\
\hline 5 & $(8,12,4,1)$ & 26 & 0.034 \\
\hline
\end{tabular}

Для того, чтобы вычислить вероятность отбора хромосомы для будущей популяции, мы можем воспользоваться формулой

$$
P_{i}=\frac{O \Pi_{i}}{\sum_{i=1}^{5} O \Pi_{i}}
$$

Результаты использования этой формулы показаны в табл. 2.

Таблицุа 2.

Вероятность отбора хромосом

\begin{tabular}{|c|c|c|}
\hline Номер $\mathrm{i}$ & $\mathrm{P}_{\mathrm{i}}$ & $\mathrm{U}_{\mathrm{i}} \%$ \\
\hline 1 & $0.013 / 0.135=0.096$ & 9.6 \\
\hline 2 & $0.039 / 0.135=0.288$ & 28.8 \\
\hline 3 & $0.037 / 0.135=0.274$ & 27.4 \\
\hline 4 & $0.0071 / 0.135=0.05259$ & 5.259 \\
\hline 5 & $0.034 / 0.135=0.2518$ & 25.18 \\
\hline
\end{tabular}

Для того, чтобы сделать дальнейший выбор хромосом мы воспользуемся методом колеса рулетки. При этом для каждой из хромосом мы ставим в соответствие сектор на колесе $\mathrm{U}_{\mathrm{i}}$ (рис. 4).

После того, как произошло каждое вращение колеса, происходит выбор какого-то сектора, то есть хромосомы. После того, как произошло 15 вращений колеса, отбираются 15 хромосом, из них на основе случайного закона происходит формирование 5 пар для того, чтобы осуществлялось скрещивание. Результаты расчетов приведены в табл. 3. 


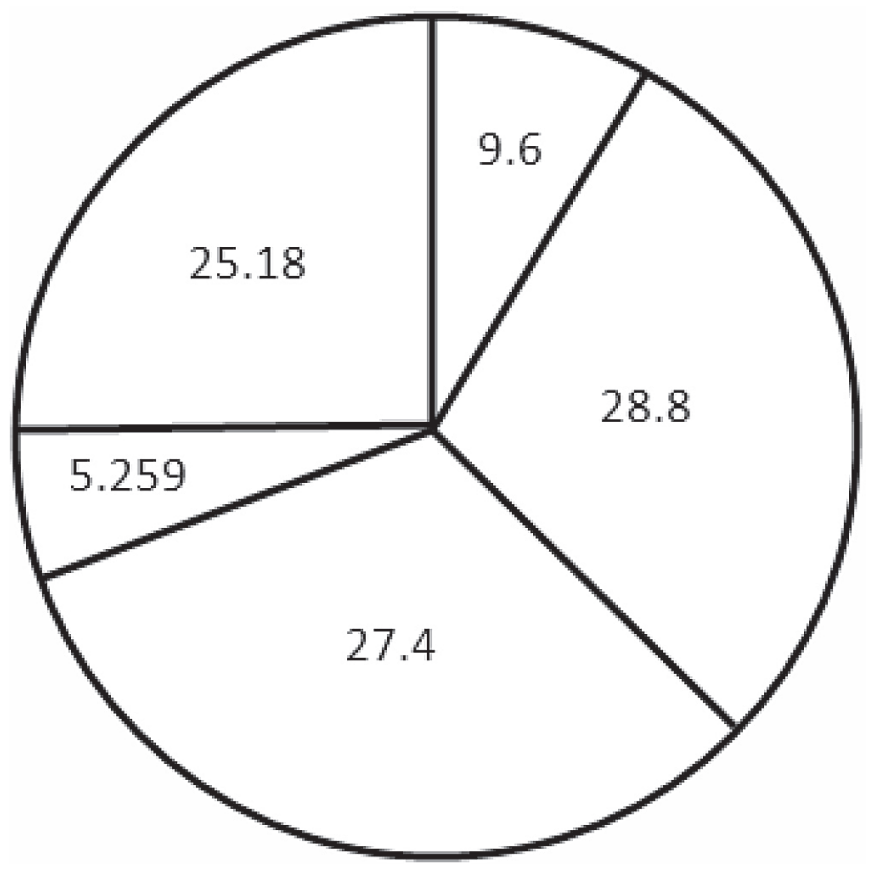

Рис. 4. Распределение секторов рулетки

Таблица 3.

Результаты отбора на основе метода колеса рулетки

\begin{tabular}{|c|c|}
\hline Номер і отца & Номер і матери \\
\hline 2 & 2 \\
\hline 4 & 3 \\
\hline 2 & 5 \\
\hline 3 & 4 \\
\hline 4 & 4 \\
\hline
\end{tabular}

В соответствии с табл. 2 самую плохую хромосому 4 мы ни разу отобрали для скрещивания. Хромосому 1 мы выбирали для скрещивания всего один раз, а хромосомы 2, 3и 5 мы отбирали часто, так как им соответствует высокая ОП. 
Следующей генетической операцией является скрещивание. Средней ошибкой решения для популяции потомков является 37 , Но для первоначальной популяции значение этого коэффициента было 57.

Рассмотренный подход может быть использован для широкого класса электродинамических структур [13-17].

В результате оптимизации нами было установлено, что для частоты 10 ГГц при угле наблюдения $30^{\circ}$ величина уровня эффективной площади рассеяния не превышает 20 дБ при размерах элемента периодической структуры $1.5 \lambda$ и расстоянии между элементами 3.2 $\lambda$.

Вывод. В работе проведено моделирование рассеяния электромагнитных волн на электродинамической периодической структуре. Рассмотрен алгоритм оптимизации (генетический алгоритм), с использованием которого возможно определение параметров электродинамической структуры, при которых достигаются требуемые значения характеристик рассеяния для заданного угла наблюдения.

\section{Сиисок литературы}

1. Головинов С.О., Хромых А.А. Проблемы управления системами мобильной связи // Вестник Воронежского института высоких технологий. 2012. № 9. С. 13-14.

2. Казаков Е.Н. Разработка и программная реализации алгоритма оценки уровня сигнала в сети wifi // Моделирование, оптимизация и информационные технологии. 2016. № 1. С. 13.

3. Пекшев Г.А., Скляр А.Г. Некоторые свойства лучевых методов, используемых для анализа распространения электромагнитных волн // В мире научных открытий. 2015. № 12. С. 17-23.

4. Пекшев Г.А., Скляр А.Г. Проблемы управления рассеянными электромагнитными полями // В мире научных открытий. 2015. № 12. С. 24-30. 
5. Максимова А.А., Юрочкин А.Г. Методы исследования характеристик рассеяния электромагнитных волн объектами // Вестник Воронежского института высоких технологий. 2016. № 16. C. 53-56.

6. Кеванишвили Г.Ш. О дифракции плоской электромагнитной волны на решетке, составленной из прямоугольных пластинок / Г.Ш. Кеванишвили, Д.К. Квавадзе, П.И. Бекаури // Радиотехника и электроника, 1966. Т. 11. № 1. С. 136-139.

7. Глотова Т.В. Применение гибридного метода для расчета характеристик рассеяния объектов над шероховатой поверхностью // Моделирование, оптимизация и информационные технологии. 2016. № 1. С. 11.

8. Щербатых С.С. Метод интегральных уравнений как основной способ анализа в САПР антенн // Моделирование, оптимизация и информационные технологии. 2016. № 1. С. 10.

9. Болучевская О.А., Горбенко О.Н. Свойства методов оценки характеристик рассеяния электромагнитных волн // Моделирование, оптимизация и информационные технологии. 2013. № 3. С. 4.

10. Вычислительные методы в электродинамике / Под ред. Р. Митры. М.: Мир, 1977. 485 с.

11. Гладков Л.А., Курейчик В.В., Курейчик В.М. Генетические алгоритмы. М.: Физматлит, 2006. 320 с.

12.Ерасов С.В. Оптимизационные процессы в электродинамических задачах // Вестник Воронежского института высоких технологий. 2013. № 10. С. 2026.

13. Канавин С.В., Лукьянов А.С. Перспективы применения систем мобильного широкополосного доступа в сетях подвижной радиосвязи на основе стандартов mobile WIMAX и LTE // Вестник Воронежского института высоких технологий. 2016. № 16. С. 79-82.

14. Кульнева Е.Ю., Гащенко И.А. О характеристиках, влияющих на моделирование радиотехнических устройств // Современные наукоемкие технологии. 2014. № 52. С. 50. 
15. Баранов А.В. Проблемы функционирования mеshсетей // Вестник Воронежского института высоких технологий. 2012. № 9. С. 49-50. 16.Милошенко О.В. Методы оценки характеристик распространения радиоволн в системах подвижной радиосвязи // Вестник Воронежского института высоких технологий. 2012. № 9. С. 60-62.

17. Мишин Я.А. О системах автоматизированного проектирования в беспроводных сетях // Вестник Воронежского института высоких технологий. 2013. № 10. С. 153-156.

\section{References}

1. Golovinov S.O., Khromykh A.A. Vestnik Voronezhskogo instituta vysokikh tekhnologiy. 2012. № 9, pp. 13-14.

2. Kazakov E.N. Modelirovanie, optimizatsiya i informatsionnye tekhnologii. 2016. № 1. P. 13.

3. Pekshev G.A., Sklyar A.G. V mire nauchnykh otkrytiy. 2015. № 12, pp. 17-23.

4. Pekshev G.A., Sklyar A.G. V mire nauchnykh otkrytiy. 2015. № 12, pp. 24-30.

5. Maksimova A.A., Yurochkin A.G. Vestnik Voronezhskogo instituta vysokikh tekhnologiy. 2016. № 16, pp. 53-56.

6. Kevanishvili G.Sh., Kvavadze D.K., Bekauri P.I. Radiotekhnika $i$ elektronika, 1966. V. 11. № 1, pp. 136-139.

7. Glotova T.V. Modelirovanie, optimizatsiya i informatsionnye tekhnologii. 2016. № 1. P. 11.

8. Shcherbatykh S.S. Modelirovanie, optimizatsiya i informatsionnye tekh-nologii. 2016. № 1. P. 10.

9. Boluchevskaya O.A., Gorbenko O.N. Modelirovanie, optimizatsiya $i$ infor-matsionnye tekhnologii. 2013. № 3. P. 4.

10. Vychislitel'nye metody $v$ elektrodinamike [Numerical methods in electrodynamics]/ R. Mitry (ed.). M.: Mir, 1977. 485 p.

11. Gladkov L.A.,Kureychik V.V., Kureychik V.M. Geneticheskie algoritmy [Genetic Algorithms]. M.: Fizmatlit, 2006. 320 p. 
12.Erasov S.V. Vestnik Voronezhskogo instituta vysokikh tekhnologiy. 2013. № 10. P. 2026.

13. Kanavin S.V., Luk'yanov A.S. Vestnik Voronezhskogo instituta vysokikh tekhnologiy. 2016. № 16, pp. 79-82.

14. Kul'neva E.Yu., Gashchenko I.A. Sovremennye naukoemkie tekhnologii. 2014. № 52. P. 50.

15. Baranov A.V. Vestnik Voronezhskogo instituta vysokikh tekhnologiy. 2012. № 9, pp. 49-50.

16. Miloshenko O.V. Vestnik Voronezhskogo instituta vysokikh tekhnologiy. 2012. № 9, pp. 60-62.

17. Mishin Ya.A. Vestnik Voronezhskogo instituta vysokikh tekhnologiy. 2013. № 10, pp. 153-156.

\section{ДАННЫЕ ОБ АВТОРАХ}

\section{Преображенский А.П., Чопоров О.Н.}

Воронежский институт высоких технологий

ул. Ленина, 73А, Воронеж, Воронежская обл., 394043, Российская Федерация

komkovvivt@ya.ru

\section{DATA ABOUT THE AUTHORS \\ Preobrazhensky A.P., Choporov O.N.}

Voronezh Institute of High Technologies

73A, Lenin Str., Voronezh region, 394043, Russian Federation komkovvivt@ya.ru 